\title{
Rapid Modelling and Grinding of Workpieces' Inner-surface by Robot with Impedance Model Based Fuzzy Force Control Algorithm
}

\author{
Wang Fei , Luo Zhong and Liu Hongyi \\ School of Mechanical Engineering \& Automation, Northeastern University, 110819, Shenyang, China
}

\begin{abstract}
To achieve rapid automatic grinding of workpieces' inner-surface by industrial robot, a rapid translational detection strategy of workpieces' inner-surface and fuzzy force control algorithm of grinding are proposed in this paper. The rapid translational detection strategy introduces a way to establish an inner-surface's model quickly by recording key points of the axial section contour which reflects big curvature changes of the contour. The established model is feasible but imprecision. The force control algorithm is based on impedance model. To promote adaptability to the imprecision of the established inner-surface's model, a fuzzy adjusting strategy is introduced in the force control algorithm. By adopting an adjusting factor, which determined by force response and a fuzzy logic, the strategy can adjust the reference trajectory of impedance model in time. Taking advantage of proposed detection and force control method, grinding experiments shows that the contact normal force maintains approximately constant, the relative mean error is within $6.5 \%$, and the material removal thickness of the inner-surface is approximately consistent. The proposed strategy's feasibility is verified.
\end{abstract}

\section{Introduction}

Industrial robot is a tendency in the manufacturing process in INDUSTRIE 4.0[1]. When robot contact with a workpiece, such like grinding, deburring and polishing, compliance is necessary for the robot[2]. Moreover, the precision of workpieces' model and workpieces' location in its jig are both important to the constraint machining process.

A. Robertsson etc. established a robot platform with external fast open sensor integration system, and implemented force-controlled grinding, finishing and deburring experiments with the platform[3]. In the experiments, an off-line programming including path generation based on work-pieces' CAD model is introduced in the hybrid force/position control strategies.

Considering the position and orientation errors of the workpiece, Hee-Chan Song and Jae-Bok Song developed a tool-path modification method based on a CAD model and direct teaching. By setting a virtual wall, the force control based on impedance model is used to applying an accurate and stable contact force[4].

Accuracy of workpieces affects the performance of force control intensively. To obtain accurate model of the workpiece, an off-line pre-programming is needed in those researches. Pre-programming is obviously timeconsuming but beneficial to force control. In order to shorten the whole work time, a less-accurate model of workpieces is acceptable. Meanwhile, adaptability to the imprecision model is put forward to the force control algorithm.
To solve the above mentioned problem, a rapid translational detection strategy and fuzzy force control algorithm, which can establish a workpiece's model quickly and adapt the imprecision of the model, are proposed in this paper. Modelling and grinding experiments are described in the end of the paper.

\section{Model of the grinding robot system}

When grinding a cavity's inner-surface with a constant material removal thickness, the sketch map of the grind robot system is shown in figure1. The cavity is assumed to be a rotary shell. The robot has 5-DOF to grinding the inner-surface of the shell, including a translational joint, 2 rotational joints and a tool and a shell's rotational movement[5].

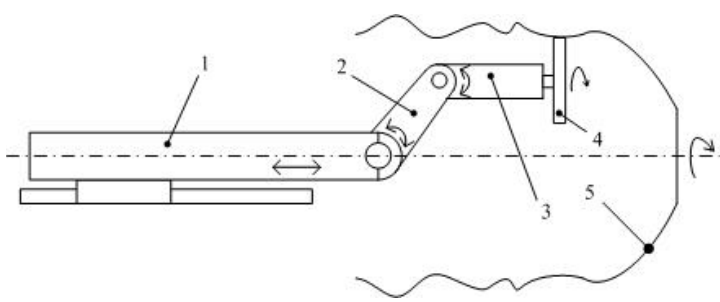

Figure 1. Sketch map of the grinding robot(1. 1st arm, 2. 2nd arm, 3. 3rd arm, 4. grinding tool, 5. rotary shell's inner-surface)

\subsection{Model of the grinding robot}


The dynamic model of an n-link rigid-joint manipulator in joint coordinate is described by

$$
\boldsymbol{M}(\boldsymbol{q}) \ddot{\boldsymbol{q}}+\boldsymbol{C}(\boldsymbol{q}, \dot{\boldsymbol{q}}) \dot{\boldsymbol{q}}+\boldsymbol{G}(\boldsymbol{q})=\boldsymbol{\tau}+\boldsymbol{J}^{\mathrm{T}} \boldsymbol{F}_{e}
$$

where $\boldsymbol{q}, \dot{\boldsymbol{q}}, \ddot{\boldsymbol{q}}$ correspond to the joints' position, velocity and acceleration vectors, respectively. $\boldsymbol{M ( q )}$ is the $\mathrm{n} \times \mathrm{n}$ positive-definite inertia matrix. $\boldsymbol{C}(\boldsymbol{q}, \dot{\boldsymbol{q}})$ is the $\mathrm{n} \times \mathrm{n}$ centripetal and Coriolis matrix. $\boldsymbol{G}(\boldsymbol{q})$ is the $\mathrm{n} \times 1$ vector of gravitational terms in workspace. $\tau$ is the $\mathrm{n} \times 1$ joint input torque. $\boldsymbol{J}$ represents the $\mathrm{n} \times 6$ Jacobian matrix. The robot dynamic model in the Cartesian space can be derived from formula(1):

$$
\boldsymbol{M}_{\mathrm{x}}(\boldsymbol{x}) \ddot{\boldsymbol{x}}+\boldsymbol{C}_{\mathrm{x}}(\boldsymbol{x}, \dot{\boldsymbol{x}}) \dot{\boldsymbol{x}}+\boldsymbol{G}_{\mathrm{x}}(\boldsymbol{x})=\boldsymbol{J}^{-\mathrm{T}} \boldsymbol{\tau}+\boldsymbol{F}_{\mathrm{e}}
$$

where $\boldsymbol{M}_{\mathrm{x}}(B)=\boldsymbol{J}^{-\mathrm{T}} \boldsymbol{M J}^{-1}, \boldsymbol{C}_{\mathrm{x}}(\boldsymbol{x}, \dot{\boldsymbol{x}})=\boldsymbol{J}^{-\mathrm{T}}\left(\boldsymbol{C}-\boldsymbol{M J}^{-1} \boldsymbol{j}\right) \boldsymbol{J}^{-1}$ and $\boldsymbol{G}_{\mathrm{x}}(\boldsymbol{x})=\boldsymbol{J}^{-\mathrm{T}} \boldsymbol{G}$.

\subsection{Contour detecting and modelling of the inner-surface}

Usually, the contour of the shell's inner-surface has great difference with its CAD model, cause the shell's crafting process. And position errors inevitably occur when a workpiece is placed in the jig, shown in figure2. The CAD model can not be used directly in robot's grinding process. Therefore, the inner-surface's model needs to be established to guarantee the accurate grinding process.

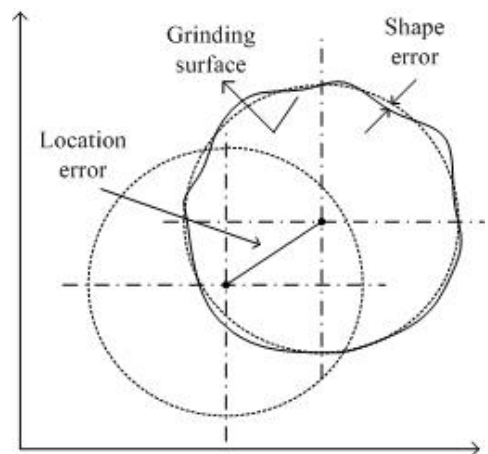

Figure 2. Location and shape error of an inner-surface's contour

\subsubsection{Rapid translational detection strategy}

When the actual contour of the shell' inner-surface has great difference with its CAD model, the model could be used as a reference only. Fix a laser sensor, displacing the grinding tool, in the end of the robot manipulator. With the CAD model as reference, detecting the innersurface's axial contour, the detection could be conducted according to the following steps.

1. Actuating the manipulator into the cavity, let the end of the manipulator locate approximately in the middle of the cavity. The serial number of this cavity's axial section represents by $j(j=0,1, \cdots, m)$.

2. Taking the CAD model as reference, let the laser beam be perpendicular to the suppositional inner-surface.
Set the orientation of the end-effector as the initial orientation. Actuate the manipulator in the initial orientation until the laser sensor got a valid data equalled to $D_{\mathrm{d}}$, which is the median of the laser sensor's measuring range. Calculate this first detection point's coordination value $P_{0}^{j}$ in the base coordinate system according to the robot's kinematics model.

3. Maintaining the end-effector's gesture, let the parallel motion of laser beam be along with one axial direction of the cavity until the sensor's output $D_{\text {out }}$ changed out of range $\Delta_{f}$. When sensor's output changed out of rang, which means $\left|D_{\text {out }}-\mathrm{D}_{\mathrm{d}}\right| \geq \Delta_{f}$, the point detected can be recorded as $P_{i}^{j}(i=1,2, \cdots t)$.

4. On the basis of maintaining the laser beam sticking on detection point $P_{i}^{j}$, adjusting end-effector's position and gesture, let laser sensor's output be equalled to $D_{\mathrm{d}}$ and laser beam be perpendicular to the line formed by $P_{i-1}^{j}$ and $P_{i}^{j}$.

5. Repeating step 3 and 4 with direction $P_{i}^{j}-P_{i-1}^{j}$ until laser sensor got no valid output, a series $\left[\begin{array}{llll}P_{0}^{j} & P_{1}^{j} & \cdots & P_{t}^{j}\end{array}\right]$ will be recorded.

6. Actuating the manipulator return to the status of the first point $P_{0}^{j}$, and repeating step 3 to 5 in the opposite direction, series $\left[\begin{array}{llll}P_{t+1}^{j} & P_{t+2}^{j} & \cdots & P_{n}^{j}\end{array}\right]$ will be recorded.

7. Resettling and combining the series $\left[\begin{array}{llll}P_{0}^{j} & P_{1}^{j} & \cdots & P_{t}^{j}\end{array}\right]$ and $\left[\begin{array}{llll}P_{t+1}^{j} & P_{t+2}^{j} & \cdots & P_{n}^{j}\end{array}\right]$ along with the cavity's axial direction, the resettled series $\left[\begin{array}{llll}P_{0}^{j} & P_{1}^{j} & \cdots & P_{n}^{j}\end{array}\right]$ will sketch an axial contour of the cavity's inner-surface, shown as figure3.

8. Rotating the cavity in a small degree and repeating step 1 to 7 , series $\left[P_{0}^{j+1} P_{1}^{j+1} \cdots P_{k}^{j+1}\right]$ will be recorded. Where, $k$, representing the quantity of recorded points, is determined by complexity of the axial contour.

9. Repeating step 1 to 8 , after the whole axial contours being detected, key points of the cavity's innersurface will form a bivector as $\boldsymbol{P}_{i}^{j}(i=1,2, \cdots k, j=1,2, \cdots m)$.

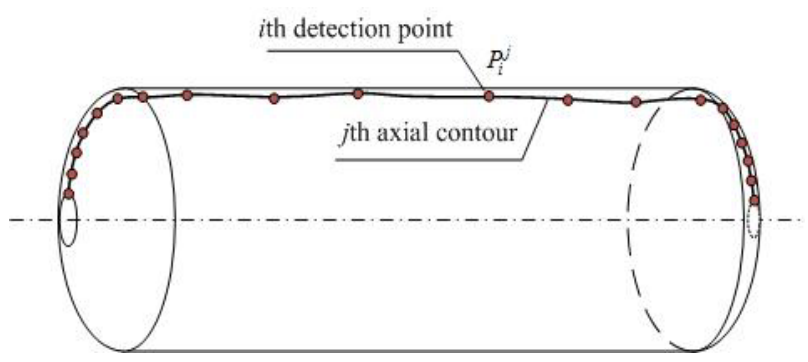

Figure 3. Detection points series of a cavity's inner-surface contour

\subsubsection{Simulation of translational detection strategy}


Setting $\Delta_{f}$ as $0.5 \mathrm{~mm}$ and $2 \mathrm{~mm}$, the detection simulation results are shown in Fig. 4 and Fig. 5.

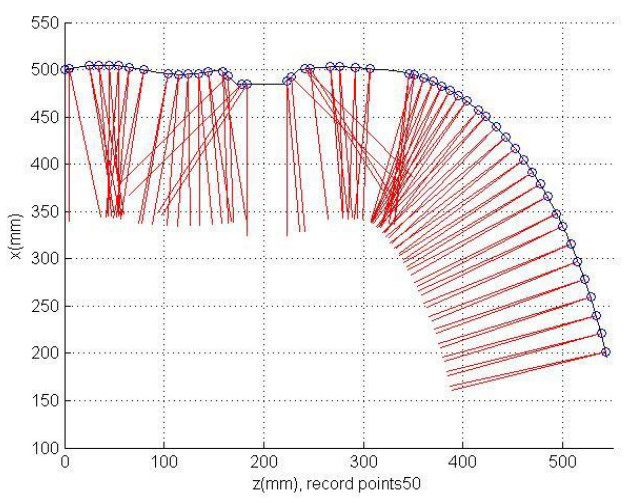

Figure 4. Detection with $\Delta_{f}=0.5 \mathrm{~mm}$

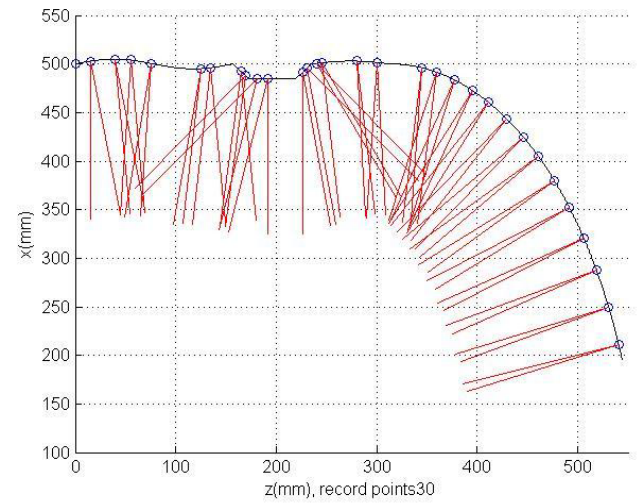

Figure 5. Detection with $\Delta_{f}=2 \mathrm{~mm}$

The simulation results shown that the translational detection strategy proposed above is feasible. Moreover, the smaller $\Delta_{f}$ is the more accurate the model goanna be. However, the model is not detailed enough. It only includes several key points that can reflect big curvature changes of the contour. Therefore, the model established by proposed rapid translational detection strategy is feasible but imprecision. An intelligent force control algorithm which can be adaptive the imprecision is needed.

\section{Force control algorithm}

Since the existing imprecision of both contour model and location of the inner-surface, the force control algorithm should be adaptive to the mentioned error. A fuzzy force control strategy based on impedance control model is proposed.

\subsection{Impedance control model}

Using the following impedance mathematic model [6]:

$$
\boldsymbol{M}_{\mathrm{d}}\left(\ddot{\boldsymbol{x}}_{\mathrm{d}}-\ddot{\boldsymbol{x}}\right)+\boldsymbol{D}_{\mathrm{d}}\left(\dot{\boldsymbol{x}}_{\mathrm{d}}-\dot{\boldsymbol{x}}\right)+\boldsymbol{K}_{\mathrm{d}}\left(\boldsymbol{x}_{\mathrm{d}}-\boldsymbol{x}\right)=\boldsymbol{F}_{\mathrm{d}}-\boldsymbol{F}_{\mathrm{e}}
$$

where $\boldsymbol{M}_{\mathrm{d}}, \boldsymbol{D}_{\mathrm{d}}$ and $\boldsymbol{K}_{\mathrm{d}}$ are respectively the desired inertia, damping and stiffness matrices of the manipulator-environment system. $\quad \boldsymbol{F}_{\mathrm{d}}$ is the desired contact force between manipulator's end-effector and the constraint surface. $\boldsymbol{x}, \dot{\boldsymbol{x}}$ and $\ddot{\boldsymbol{x}}$ are the actual position, velocity and acceleration of the robot manipulator, respectively. $\boldsymbol{x}_{\mathrm{d}}, \dot{\boldsymbol{x}}_{\mathrm{d}}$ and $\ddot{\boldsymbol{x}}_{\mathrm{d}}$ is respectively the desired trajectory, velocity and acceleration.

\subsection{Reference trajectory's fuzzy adjusting strategy}

Assuming $x_{\mathrm{e}}$ and $K_{\mathrm{e}}$ to be the actual position and stiffness of the constraint surface. $x_{\mathrm{d}}$ can be represents as:

$$
x_{\mathrm{d}}=x_{\mathrm{e}}+\frac{F_{\mathrm{d}}}{K_{\mathrm{e}}}
$$

Usually, the accurate $x_{\mathrm{e}}$ and $K_{\mathrm{e}}$ are unavailable. Let the inaccurate value $x_{\mathrm{ec}}$ and $K_{\mathrm{ec}}$ replace the $x_{\mathrm{e}}$ and $K_{\mathrm{e}}$ in formula(4). And the approximate $K_{\mathrm{ec}}$ is usually experiential.

According to the approximately rough function, the reference trajectory should be:

$$
x_{\mathrm{r}}=x_{\mathrm{ec}}+\frac{F_{\mathrm{d}}}{K_{\mathrm{ec}}}
$$

To promote adaptability of the control algorithm, an intelligent prediction and adjusting of the reference trajectory is introduced into the impedance model [7].

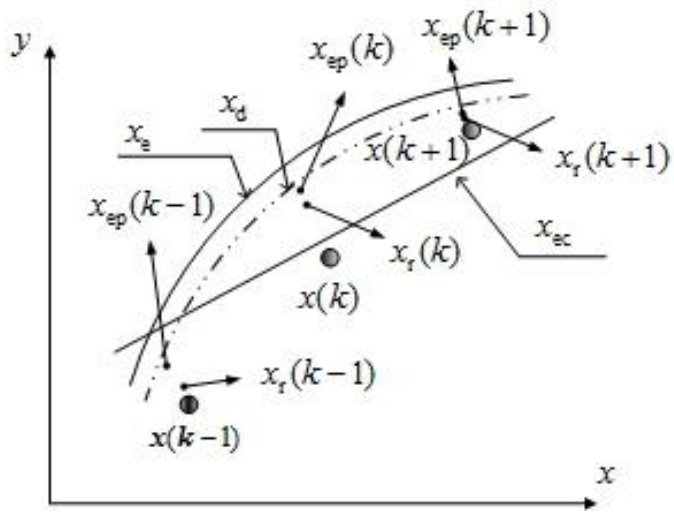

Figure 6. Reference trajectory adjusting

Establish the reference trajectory intelligent adjusting law in discrete form as:

$$
\boldsymbol{x}_{\mathrm{r}}(k+1)=\boldsymbol{x}_{\mathrm{r}}(k)+\Delta x_{\mathrm{ec}}(k)
$$

where $k$ is defined as the current moment. $\boldsymbol{x}_{\mathrm{r}}(k+1)$ is the predictive position of reference trajectories in time $\boldsymbol{k}+1 . \boldsymbol{x}_{\mathrm{r}}(k)$ is the reference trajectory in time $\boldsymbol{k}$. The term $\Delta x_{\mathrm{ec}}(k)$ is an adjustment based on the predicted location and geometric error: 


$$
\Delta x_{\mathrm{ec}}(k)=x_{\mathrm{ec}}(k+1)-x_{\mathrm{ec}}(k)+k_{\mathrm{c}} \cdot e_{\mathrm{xp}}(k)
$$

where, $\quad e_{\mathrm{xp}}(k)=\Delta x_{\mathrm{ep}}(k)-\Delta x_{\mathrm{ep}}(k-1) \quad$ represents the tendency of the adjusting error. Setting $x_{\mathrm{ep}}(k)=x(k)-\frac{F_{\mathrm{e}}(k)}{K_{\mathrm{ec}}}$, represents the approximately actual position of the constraint surface, $\Delta x_{\mathrm{ep}}(k)=x_{\mathrm{ep}}(k)-x_{\mathrm{ec}}(k)$ approximately reflects the distance from the reference trajectories to the actual surface. $k_{\mathrm{c}}$ is the adapting scale factor determined by a fuzzy controller.

The input of the fuzzy controller is $\left|e_{\mathrm{xp}}(k)\right|$.The linguistic variables assigned to $e_{\mathrm{xp}}$ and $k_{\mathrm{c}}$ are $\mathrm{EX}$ and $\mathrm{KC}$. The corresponding fuzzy subsets are $\mathrm{A}$ and $\mathrm{B}$. The set $\mathrm{A}$ comprises $\mathrm{VB}, \mathrm{B}, \mathrm{M}, \mathrm{S}$ and $\mathrm{VS}$, which respectively denote very big, big, middle, small and very small. The set $\mathrm{B}$ comprises $\mathrm{KB}, \mathrm{BK}, \mathrm{KM}, \mathrm{SK}$ and $\mathrm{KS}$, which denote very big, big, middle, small and very small respectively. The fuzzy rules for input $\mathrm{EX}$ and output $\mathrm{KC}$ are shown in Table I.

Table 1. Fuzzy Control Rules for The Output KC

\begin{tabular}{|c|c|c|c|c|c|}
\hline \multirow{2}{*}{} & \multicolumn{5}{|c|}{ EXP } \\
\cline { 2 - 6 } & VB & B & M & S & VS \\
\hline KC & KB & BK & KM & SK & KS \\
\hline
\end{tabular}

Replacing the $\boldsymbol{x}_{\boldsymbol{d}}, \dot{\boldsymbol{x}}_{\mathrm{d}}$ and $\ddot{\boldsymbol{x}}_{\mathrm{d}}$ with $\boldsymbol{x}_{\boldsymbol{r}}, \dot{x}_{\mathrm{r}}$ and $\ddot{x}_{\mathrm{r}}$ in formula(3), define $\boldsymbol{u} \equiv \ddot{\boldsymbol{x}}$ as the control rule. It could be educed as:

$$
\boldsymbol{u}=\ddot{\boldsymbol{x}}_{\mathrm{d}}+\boldsymbol{M}_{\mathrm{d}}^{-1}\left(\boldsymbol{D}_{\mathrm{d}} \dot{\boldsymbol{e}}_{\mathrm{r}}+\boldsymbol{K}_{\mathrm{d}} \boldsymbol{e}_{\mathrm{r}}-\boldsymbol{F}_{\mathrm{d}}+\boldsymbol{F}_{\mathrm{e}}\right)
$$

where, $\boldsymbol{e}_{\mathrm{r}}=\boldsymbol{x}_{\mathrm{r}}-\boldsymbol{x}, \dot{\boldsymbol{e}}_{\mathrm{r}}=\dot{\boldsymbol{x}}_{\mathrm{r}}-\dot{\boldsymbol{x}}$ and $\ddot{\boldsymbol{e}}_{\mathrm{r}}=\ddot{\boldsymbol{x}}_{\mathrm{r}}-\ddot{\boldsymbol{x}}$.

Combining formula(1) and (8), the join torque control law of the force control model with reference trajectory online intelligent adjusting could be:

$$
\boldsymbol{\tau}=\boldsymbol{J}^{\mathrm{T}}\left[\boldsymbol{M}_{\mathrm{x}} \boldsymbol{u}+\boldsymbol{C}_{\mathrm{x}} \dot{\boldsymbol{x}}+\boldsymbol{G}_{\mathrm{x}}-\boldsymbol{F}_{\mathrm{e}}\right]
$$

\subsection{Impedance model based force control}

The control law is shown in Fig. 3. When robot's in a constraint motion, the impedance based intelligent force controller will be implemented to perform a constant force between the robot end-effector and constraint surfece.

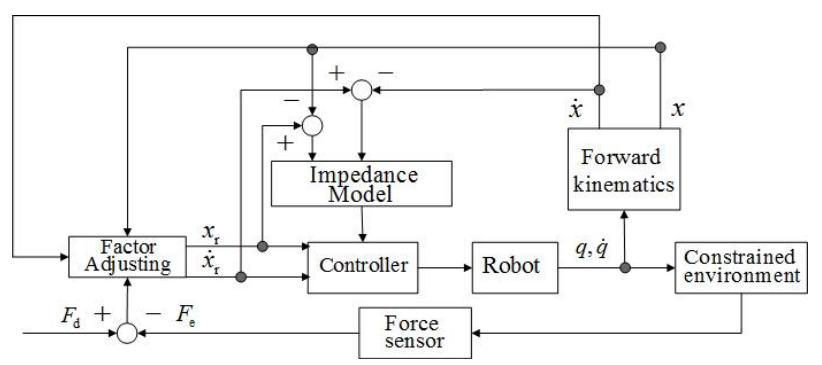

Figure 7. Scheme of the impedance model based force control

\section{Detecting and grinding experiment}

The grinding robot system is shown in Fig. 8. The CAD model of cavity's inner-surface is available but has a great difference with the actual object. Firstly detecting the inner-surface of the shell with a laser sensor, the 3D model of the inner-surface could be built as figure 9 . Then executing the grinding process based on the proposed fuzzy force control algorithm, with an expected contact force $5 \mathrm{~N}$, the force response is shown in figure 10 .

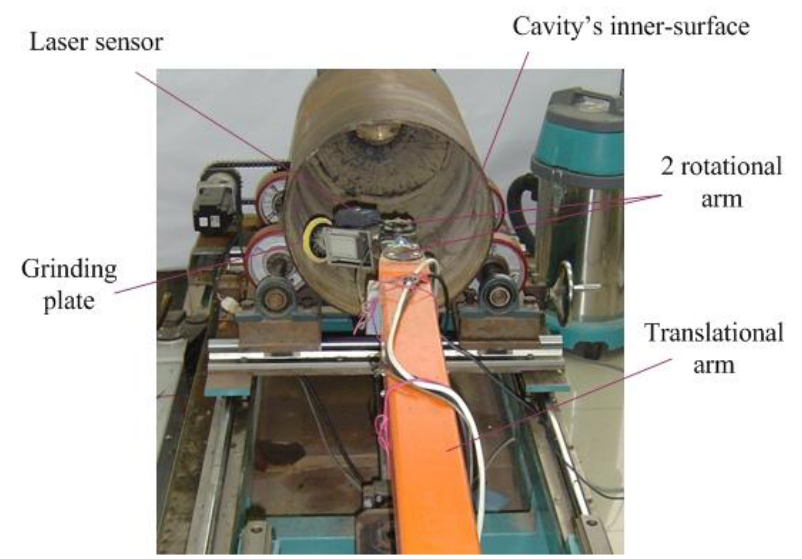

Figure 8. Grinding experiment of a rotary shell's inner-surface

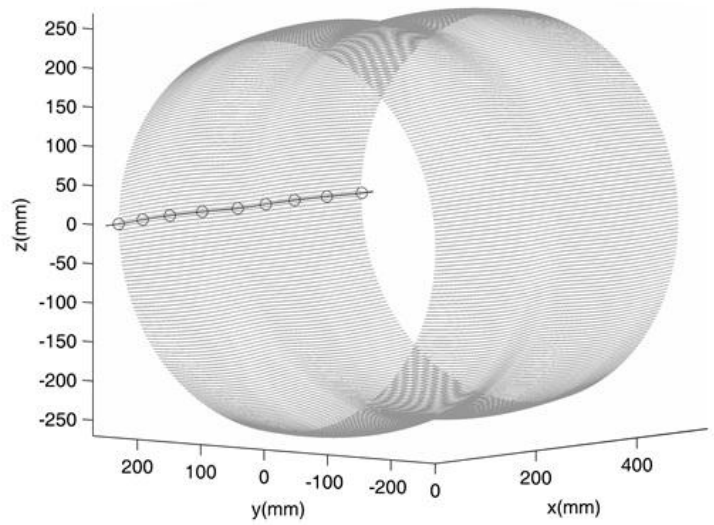

Figure 9. Reconstructed 3D model of the rotary shell's inner-surface

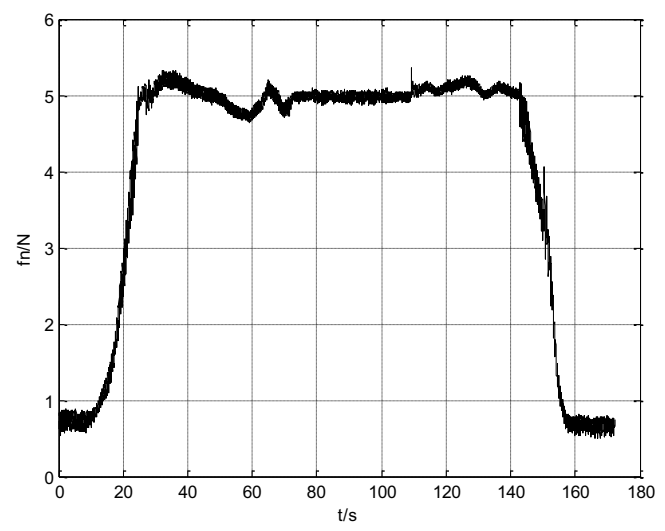

Figure 10. Normal force response with expected $5 \mathrm{~N}$

Fig. 10 shows that the contact normal force between manipulator's end-effector and the constrained innersurface is controlled to be around its expected value. The 
fluctuation in the response is caused by inaccurate model of the inner-surface and impact of the manipulator's movement from free movement to a restraint movement in the very beginning of the grinding process. Moreover, when grinding process is in time 50 70s, there's fluctuation because that the curvature of the constrained surface is very small and changed drastically. Taking advantage of proposed fuzzy force control strategy, error of contact normal force fluctuate in $[-0.3,0.2] \mathrm{N}$, with relative error within $5 \%$. Overall, in the whole grinding process, the relative mean error of normal contact force from the expected value is within $6.5 \%$. The material removal thickness of the inner-surface is approximately consistent.

\section{Conclusion}

To grind a cavity's inner-surface automatically with robot, a rapid translational detection strategy and fuzzy force control algorithm are proposed in this paper. A compendious model could be established quickly by following the rapid translational detection strategy which developed to record key points of the inner-surface's contour. Taking advantage of the fuzzy force control algorithm based on impedance control algorithm, the material removal thickness in grinding process can be controlled to be approximately consistent. The force control algorithm is adaptive to the contour model's imprecision, and maintain the contact force be approximately constant equal to the expect value with relative mean error $6.5 \%$. The experience of this research provides an important reference for the practical application.

\section{References}

1. J. F. Wan, H. Cai, K. L. Zhou. 2015 ICIT, 135(2015)

2. Y. Shiote, J. Akiyama, H. Yoshida, Y. Harada. Robotics and Automation, IEEE International Conference on Robotics and Automation, 1, 788(1995)

3. A. Robertsson, T. Olsson, R. Johansson, etc.. Proceedings of the 2006 IEEE/RSJ International Conference on Intelligent Robots and Systems, 2743(2006)

4. H. C. Song, J. B. Song. International Journal of Precision Engineering \& Manufacturing, 14(1), 85(2013)

5. F. Wang,H. Y. Liu, Z. Luo. Optics and Precision Engineering, 21(6), 1479(2013)

6. M. K. Vukobratovi, A. G. Rodic. Journal of Intelligent and Robotic Systems, 18(2), 191(1997)

7. F. Wang, Z. Luo, H. Y. Liu, L. Wang. 2010ROBIO, 1555(2010) 